\title{
Two-stage surgical approach for ruptured Salmonella aortitis
}

\author{
Zachary E. Brewer, MD, Michael D. Dake, MD, and Michael P. Fischbein, MD, PhD, Stanford, Calif
}

\author{
From the Department of Cardiothoracic Surgery, Stanford University, School of Medicine, Stanford, Calif. \\ Disclosures: Authors have nothing to disclose with regard to commercial support. \\ Received for publication Aug 6, 2017; revisions received Sept 8, 2017; accepted for publication Sept 22, 2017; \\ available ahead of print Oct 28, 2017. \\ Address for reprints: Michael P. Fischbein, MD, PhD, Department of Cardiothoracic Surgery, Stanford University \\ School of Medicine, Falk CVRB, 300 Pasteur Dr, Stanford, CA 94305 (E-mail: mfischbe@stanford.edu). \\ J Thorac Cardiovasc Surg 2018;155:e87-9 \\ $0022-5223 / \$ 36.00$ \\ Copyright (c) 2017 by The American Association for Thoracic Surgery \\ https://doi.org/10.1016/j.jtcvs.2017.09.102
}

Salmonella aortitis is an infrequent cause of abdominal and rarely, thoracic aortic aneurysm formation, with poor clinical outcomes unless treated surgically. ${ }^{1,2}$ The optimal treatment strategy remains controversial, and the area of contention includes early endovascular repair combined with antibiotic therapy versus open surgery. We report here a case of a descending thoracic aortic rupture from Salmonella aortitis. The patient was treated on an emergency basis with an endovascular stent graft (by thoracic endovascular aortic repair), followed by definitive open surgical repair after resolution of infection.

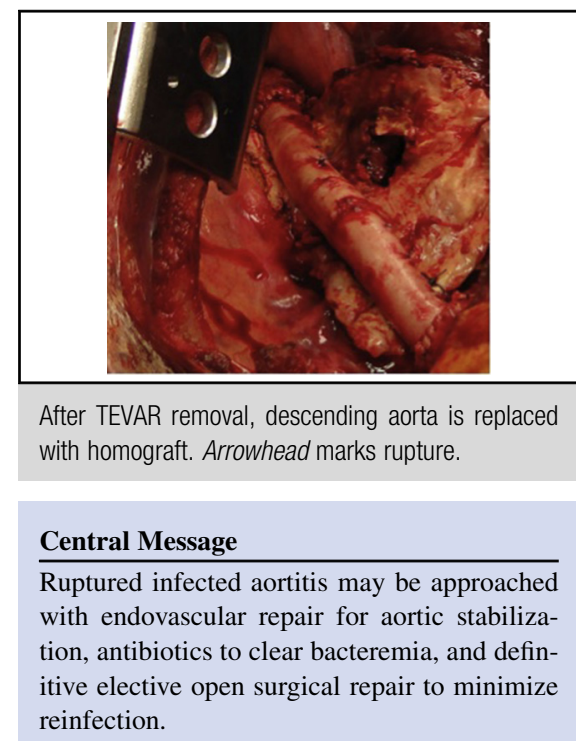

See Editorial Commentaries pages e91 and e93.

\section{CLINICAL SUMMARY}

A 70-year-old man with hypertension sought treatment at an outside hospital with a febrile illness and was discharged home after a positive test result for influenza. The patient returned 3 days later with hemoptysis, severe chest pain, and a computed tomographic scan that revealed an anterior rupture of a descending thoracic saccular aneurysm $(7.4 \mathrm{~cm})$ with air within the hematoma, suggesting a mycotic aneurysm (Figure 1). The patient was transferred to our institution in hemodynamically unstable condition and underwent a temporizing emergency thoracic endograft with a $34 \mathrm{~mm} \times 15 \mathrm{~cm}$ Gore C-TAG prosthesis (W. L. Gore and Associates, Flagstaff, Ariz), bridging the point of rupture and extending to normal-appearing aorta proximally and distally. A computed tomographic scan after the endografting demonstrated proper positioning of the prosthesis with no evidence of endoleak. After the procedure, the patient became febrile to $39.2^{\circ} \mathrm{C}$ and had leukocytosis develop. We commenced empiric antibiotic therapy; when blood cultures from the outside hospital subsequently grew Salmonella enterica, the antibiotic regimen was switched to intravenous ceftriaxone. After a 10-day antibiotic course and negative results of blood cultures, the patient went to the operating room for definitive treatment.

After induction of general anesthesia, a double-lumen tube was placed. Neuromonitoring was used. A left anterolateral thoracotomy was performed. Partial cardiopulmonary bypass was instituted through the femoral vessels, with the patient staying warm systemically and the heart beating. A proximal clamp was placed distal to the left subclavian artery, and a distal clamp was placed at the level of the diaphragm. The descending aorta was opened, and the endograft was removed. A large amount of thrombus was evacuated, tissue was sent for culture, and a large medial aortic perforation was revealed (Figure 2). The area was débrided and irrigated with antibiotic solution. With a descending aorta homograft, the distal anastomosis was initially performed; the clamp was moved to the homograft, and flow was reestablished to a large pair of intercostal arteries (T10) though the pump retrograde. The proximal anastomosis was next performed, the graft was deaired, and both clamps were removed. Flexible bronchoscopy excluded the presence of an aortopulmonary fistula. The thoracotomy was closed in the usual fashion. Despite negative blood cultures after preoperative antibiotic therapy, intraoperative tissue cultures subsequently grew Salmonella enterica. The patient otherwise had an uneventful recovery, and he was discharged home with 6 weeks of intravenous antibiotic therapy per infectious disease physician recommendations. Unfortunately, the patient was involved in a motor vehicle accident approximately 1 month after discharge. Cardiopulmonary resuscitation was performed on the scene of the accident, with the return of spontaneous circulation, and the patient 

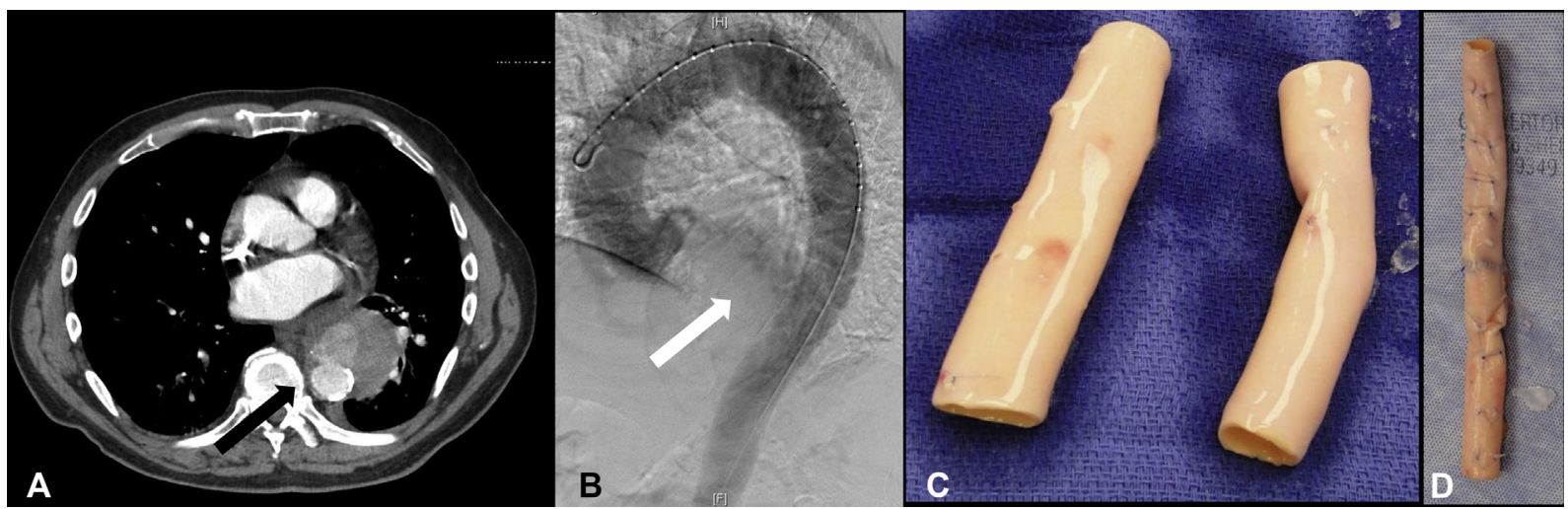

FIGURE 1. A, Axial preoperative computed tomographic image with arrow pointing to contained aortic rupture from Salmonella aortitis. B, Preoperative aortogram with arrow pointing to anterior leakage of contrast from the aortic lumen. C, Macroscopic view of 2 cryopreserved aortic homografts sutured together to create a replacement conduit of adequate length. D, Completed replacement conduit constructed from 2 aortic homografts with all intercostal branches ligated.

was transported to the hospital on an emergency basis. There, the patient arrived in extremis and repeatedly lost pulses, requiring more cardiopulmonary resuscitation. Despite aggressive resuscitation, the patient did not survive. Computed tomographic imaging demonstrated mediastinal hematoma without contrast extravasation; however, the etiology remains unclear. It is unclear if the mediastinal hematoma was a result of the motor vehicle crash, occurred before the motor vehicle crash, possibly leading to it, or was caused by cardiopulmonary resuscitation.

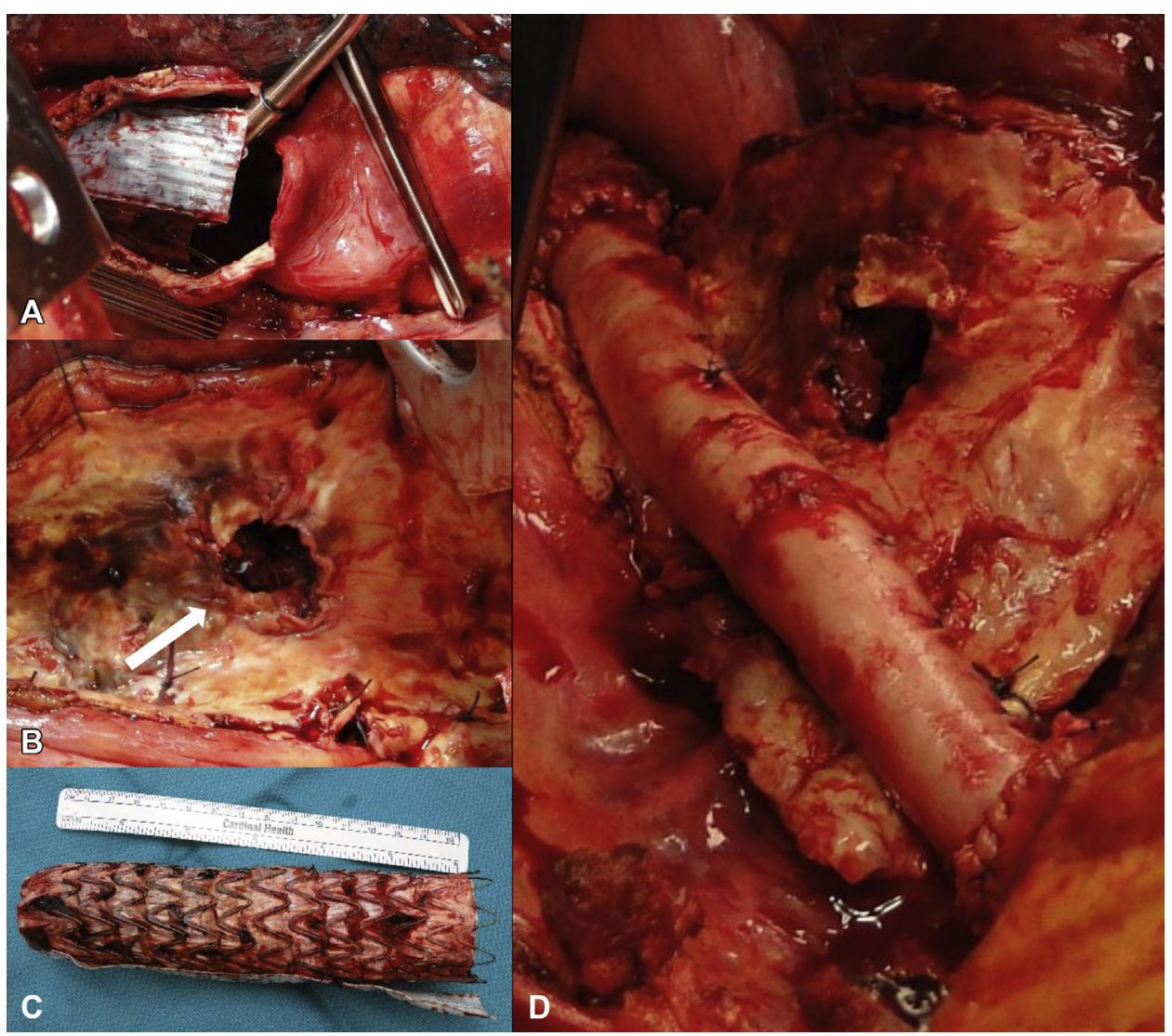

FIGURE 2. A, Descending aorta opened, demonstrating the thoracic aortic endograft. B, Descending thoracic aorta opened, with arrow pointing to site of transmural aortic rupture secondary to Salmonella aortitis. C, Explanted thoracic aortic endograft. D, Completed aortic replacement with homograft, encompassing most of the descending thoracic aorta to just above the diaphragm. 


\section{DISCUSSION}

Salmonella species are gram-negative bacteria that rarely lead to infectious aortitis, potentially progressing into aneurysm formation. Salmonella vascular infections predominately involve the abdominal aorta ( $88 \%$ of cases), but they have been reported in the thoracic aorta and coronary arteries. ${ }^{1-3}$ Because medical management results in mortalities as high as $36 \%,{ }^{3}$ surgery remains the treatment of choice. Recently. there have been encouraging short- and mid-term results with early endovascular repair in conjunction with intravenous antibiotic therapy. ${ }^{3,4}$ Of note, the duration of antibiotic therapy remains controversial, with some investigators advocating a limited duration and others recommending lifelong suppressive therapy. ${ }^{1,2}$

In this case report, despite negative blood cultures after a 10-day course of intravenous antibiotics, the intraoperative tissues remained positive for Salmonella. With mycotic aneurysms, the well-known short-term benefits of thoracic endovascular aortic repair may be outweighed by the long-term disadvantages surrounding the lack of surgical débridement of infected tissue. Forbes and Harding $^{5}$ described 2 cases of endovascular repair of Salmonella-infected aneurysms that resulted in aortoenteric fistulas, as well as recurrent sepsis. ${ }^{5}$ Although a single case, our intraoperative positive tissue cultures suggest that at least long-term suppressive antibiotics are imperative with an endovascular approach.

Homograft cryopreserved tissues have been used extensively in the setting of endocarditis and root abscesses, because they have greater resistance to recurrent infection with the absence of prosthetic material. ${ }^{6,7}$ Aortic root homografts have become less popular, however, because of lack of immediate availability, surgeon unfamiliarity and implantation difficulty, and recent studies showing no significant benefit compared with other prostheses. ${ }^{8,9}$ For the descending thoracic aorta, homografts are much easier to use technically but may require sewing multiple grafts end to end, depending on length requirements. In our case, availability was not an issue because of the need to wait for bacteremia clearance.
We have presented a case of Salmonella aortitis in which endovascular repair was used for immediate aortic stabilization to prevent free rupture and death, antibiotic therapy was used to clear bacteremia, and then definitive elective open surgical repair was finally performed. Because surgical débridement and aortic homograft use minimize any further risk of reinfection, our infectious disease team recommended a 6-week course of intravenous antibiotics. The concept of in situ reconstruction with an aortic homograft and short-term antibiotic therapy seems more acceptable than reconstruction with a synthetic graft and prolonged or even lifelong antibiotic therapy. ${ }^{7}$ This treatment paradigm would be more convincing with a larger clinical series and extended follow-up, although this is obviously unlikely because of the disease's rarity.

To our knowledge, this is the first use of a 2-staged approach for stabilization and then definitive treatment of a case of Salmonella aortitis, demonstrating the temporizing utility of endovascular stent grafting as well as the therapy of surgical excision and homograft placement.

\section{References}

1. Soravia-Dunand VA, Loo VG, Salit IE. Aortitis due to Salmonella: report of 10 cases and comprehensive review of the literature. Clin Infect Dis. 1999;29: $862-8$.

2. Lopes RJ, Almeida J, Dias PJ, Pinho P, Maciel MJ. Infectious thoracic aortitis: a literature review. Clin Cardiol. 2009;32:488-90.

3. Strahm C, Lederer H, Schwarz EI, Bachli EB. Salmonella aortitis treated with endovascular aortic repair: a case report. J Med Case Rep. 2012;6:243.

4. Montrivade S, Kittayarak C, Suwanpimolkul G, Chattranukulchai P Emphysematous Salmonella aortitis with mycotic aneurysm. BMJ Case Rep. 2017;2017:https://doi.org/10.1136/bcr-2017-220520.

5. Forbes TL, Harding GE. Endovascular repair of Salmonella-infected abdomina aortic aneurysms: a word of caution. J Vasc Surg. 2006;44:198-200.

6. Vogt PR, von Segesser LK, Goffin Y, Pasic M, Turina M. Cryopreserved arterial homografts for in situ reconstruction of mycotic aneurysms and prosthetic graft infection. Eur J Cardiothorac Surg. 1995;9:502-6.

7. Pasic M. Mycotic aneurysm of the aorta: evolving surgical concept. Ann Thorac Surg. 1996;61:1053-4

8. Kim JB, Ejiofor JI, Yammie M, Camuso JM, Walsh CW, Ando M, et al Are homografts superior to conventional prosthetic valves in the setting of infective endocarditis involving the aortic valve? J Thorac Cardiovasc Surg. 2016;151:1239-46.

9. Jassar AS, Bavaria JE, Szeto WY, Moeller PJ, Maniaci J, Milewski RK, et al Graft selection for aortic root replacement in complex active endocarditis: does it matter? Ann Thorac Surg. 2012;93:480-7. 